\title{
FORMS OF MACLAURIN'S THEOREM
}

\author{
By F. H. JACKSON.
}

[Received September 5th, 1903.-Read November 12th, 1903.-Revised January 14th, 1904.]

1. Suppose that $f(x)$ is capable of expansion in a series of the form

$$
A_{0}+A_{1} x^{[1]}+A_{2} x^{[2]}+\ldots+A_{m} x^{[m]}+\ldots,
$$

in which $[1]=p_{1},[2]=p_{1}+p_{2},[3]=p_{1}+p_{2}+p_{3}$, and in general

$$
[n]=p_{1}+p_{2}+p_{3}+\ldots+p_{n},
$$

namely, the sum of the first $n$ elements in an infinite sequence $\left(p_{1}, p_{2}, p_{3}, \ldots\right)$; then

$$
f(x)=f(0)+f^{(1)}(0) x^{[1]}+f^{(2)}(0) \frac{p_{2} !}{(2) !} x^{[2]}+\ldots+f^{(m)}(0) \frac{p_{m} !}{(m) !} x^{[m]}+\ldots
$$

The notation is as follows:

$$
\begin{gathered}
(n)=p_{1}+p_{2}+p_{3}+\ldots+p_{n}, \\
(n-1)=p_{2}+p_{3}+\ldots+p_{n}=[n]-[1], \\
(n-2)=p_{3}+p_{4}+\ldots+p_{\prime \prime}=[n]-[2], \\
(n) !=\left\{p_{1}+p_{2}+\ldots+p_{n}\right\}\left\{p_{2}+p_{3}+\ldots+p_{n}\right\} \ldots\left\{p_{n-1}-p_{n}\right\}\left\{p_{n}{ }^{\prime},\right. \\
p_{n} !=p_{1} p_{2} p_{3} \ldots p_{n}, \quad f^{(n)}(x)=D^{(n)}\{f(x)\},
\end{gathered}
$$

$D^{(n)}$ denotes the operator

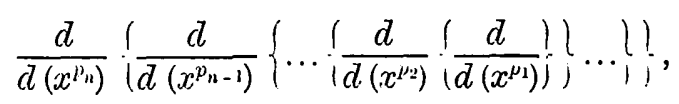

which reduces when $p_{1}=p_{2}=p_{3}=\ldots=1$ to the operator $(d / d x)^{r}$, and when the sequence of elements is $\left(1, p, p^{2}, p^{3}, \ldots\right)$ to the operator $D^{i r \bar{j}}$ (supra, pp. 84 et seq.). Examples of the theorem are afforded by Gauss's series

$$
1+\sum_{n=1}^{n=\infty} x^{\underline{n}[n(n+1)]} \quad(x<1), \quad\left(p_{1}, p_{2}, p_{3}, \ldots\right) \equiv(1,2,3,4, \ldots),
$$

and Euler's celebrated series

$\sum_{n=-\infty}^{n=+\infty}(-1)^{n} x^{1\left(3 n^{8}+1\right)}(x<1), \quad\left(p_{1}, p_{2}, p_{3}, \ldots\right)=(1,1,3,2,5,3, \ldots, 2 r-1, r, \ldots)$. 
2. If we operate on $x^{[m]}$ with $\frac{d}{d\left(x^{p_{1}}\right)}$, we obtain

$$
\frac{p_{1}+p_{2}+p_{3}+\ldots+p_{m}}{p_{1}} x^{p_{2}+p_{3}+\ldots+p_{m}} \text {. }
$$

Operating on this with $\frac{d}{d\left(x^{12}\right)}$, we obtain

$$
\frac{p_{1}+p_{2}+p_{3}+\ldots+p_{n}}{p_{1}} \frac{p_{2}+p_{9}+\ldots+p_{n}}{p_{2}} x^{p_{3}+p_{4}+\ldots+p_{m}}
$$

and in general $D^{(r)}\left\{x^{[m]}\right\}$ gives us $\frac{(m)(m-1)(m-2) \ldots(m-r+1)}{p_{1} p_{2} p_{3} \ldots p_{r}} x^{(m-r)}$; so that $D^{(m)}\left\{x^{[m]}\right\}$ gives $(m) ! / p_{m} !$.

Suppose $f(x)$ capable of expansion in a convergent series

$$
f(x)=A_{0}+A_{1} x^{[1]}+A_{2} x^{[2]}+\ldots+A_{m} x^{[m]}+\ldots
$$

If we now operate in succession with $D^{(1)}, D^{(2)}, D^{(3)}, \ldots, D^{(r)}, \ldots$, we ohtain

$$
\begin{aligned}
& \left.f^{(1)}(x)=A_{1}+A_{2} \frac{(2)}{p_{1}} x^{p_{2}}+A_{3} \frac{(3)}{p_{1}} x^{p_{2}+p_{3}}+\ldots+A_{m} \frac{(m)}{p_{1}} x^{p_{2}+p_{3}+\ldots+p_{m}}+\ldots\right) \\
& f^{(2)}(x)=A_{2} \frac{(2)(1)}{p_{1} p_{2}}+\ldots+A_{m} \frac{(m)(m-1)}{p_{1} p_{2}} x^{p_{3}+\ldots+p_{m}}+\ldots
\end{aligned}
$$

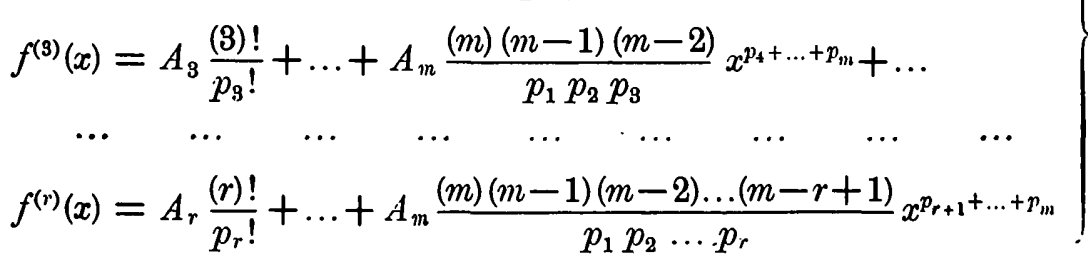

Putting $x=0$, we have

$$
A_{0}=f(0), \quad A_{1}=\frac{p_{1} !}{(1) !} f^{(1)}(0), \quad \ldots, \quad A^{(r)} \frac{\ddot{p_{r}} !}{(r) !} f^{(r)}(0) ;
$$

so that

$$
f(x)=f(0)+\frac{p_{1} !}{(1) !} f^{(1)}(0) x^{[1]}+\ldots+\frac{p_{m} !}{(m) !} f^{(m)}(0) x^{[m]}+\ldots
$$

3. In the special case when $p_{1}=p_{2}=p_{3}=\ldots=1$ we see that $p_{m} !=p_{1} p_{2} \ldots p_{m}=1, \quad(m) !=m(m-1)(m-2) \ldots 3.2 .1=m !$ and the expression (6) reduces to the normal form of Maclaurin's series. If, however, $\left(p_{1}, p_{2}, p_{3}, \ldots, p_{n}, \ldots\right)=(1,2,3, \ldots, n, \ldots)$, then

$$
p_{n} !=1.2 .3 \ldots m=m !
$$


and

$$
\begin{aligned}
(m) ! & =\{1+2+3+\ldots+m\}\{2+3+4+\ldots+m\} \ldots\{m-1+m\}\{m\} \\
& =\frac{m(m+1)}{2} \frac{(m-1)(m+2)}{2} \ldots \frac{1.2 m}{2}=\frac{2 m !}{2^{m}}
\end{aligned}
$$

and the theorem is

$$
f(x)=f(0)+f^{(1)}(0) x+f^{(2)}(0) \frac{2 ! 2^{2}}{4 !} x^{3}+\ldots+f^{(n)}(0) \frac{m ! 2^{m}}{2 m !} x^{1(m, m+1)}+\ldots
$$

The function due to Gauss

$$
\frac{1-x^{2} \cdot 1-x^{4} .1-x^{6} \ldots \text { ad inf. }}{1-x \cdot 1-x^{3} .1-x^{5} \ldots \text { ad inf. }}=1+\sum_{m=1}^{m=\infty} x^{1 .[m(m+1)]} \quad(x<1)
$$

comes under form (7), and, if we consider the series

$$
1+x^{(1)}+x^{(2)}+\ldots+x^{(n)}+\ldots
$$

as a fundamental form to which the series

$$
1+x+x^{2}+\ldots+x^{n}+\ldots=\frac{1}{1-x}
$$

and

$$
1+x+x^{3}+\ldots+x^{3(n . n+1)}+\ldots=\frac{1-x^{2} \cdot 1-x^{3} \ldots \text { ad inf. }}{1-x .1-x^{3} \ldots \text { ad inf. }}
$$

belong, we may look upon the functions (10) and (11) as analogues for the sequences $(1,1,1,1, \ldots)$ and $(1,2,3,4, \ldots)$ respectively.

4. Consider the sequence

$$
\left(p_{1}, p_{2}, p_{3}, \ldots, p_{n}, \ldots\right)=(1,3,5, \ldots, 2 n-1, \ldots) ;
$$

then the general coefficient $f^{(m)}(0) \frac{p_{m} !}{(m) !}$ becomes

$$
\begin{gathered}
f^{(m)}(0) \frac{1.3 \cdot 5 \cdot 7 \ldots 2 m-1}{m^{2} \cdot m^{2}-1^{2} \cdot m^{2}-2^{2} \ldots 2 m-1}=\frac{f^{(m)}(0)}{2^{m-1} \cdot m !} \\
f(x)=f(0)+f^{(1)}(0) x+f^{(2)}(0) \frac{x^{4}}{2 ! 2^{2}}+\ldots+f^{(m)}(0) \frac{x^{m}}{m ! 2^{m-1}}+\ldots .
\end{gathered}
$$

I have not been able to discover any II function capable of expansion in this form, but it would seem natural to expect that the ordinary functions of analysis such as $e^{x}, \sin x, \log (1-x), \& c$., whose expansions are for the simple sequence $(1,1,1, \ldots)$, would have their analogues in other sequences such as $(1,3,5, \ldots, 2 n-1)$. 
Consider the sequence $\left(p_{1}, p_{2}, p_{3}, \ldots, p_{n}, \ldots\right)=\left(1, p, p^{2}, \ldots, p^{n-1}, \ldots\right)$. Here

$$
\begin{aligned}
(m) & =1+p+p^{2}+\ldots+p^{m-1}=\frac{p^{m}-1}{p-1}=[m], \\
(m-1) & =p+p^{2}+\ldots+p^{m-1}=\frac{p^{m-1}-1}{p-1}=p[m-1], \\
f(x) & =f(0)+f^{(1)}(0) x^{[1]}+\ldots+f^{(m)}(0) \frac{x^{[m]}}{[m] !}+\ldots
\end{aligned}
$$

I have discussed series and functions of this type in the papers noted below.* Euler's theorem

$$
(1-x)\left(1-x^{2}\right)\left(1-x^{9}\right) \ldots \text { ad inf. }=\sum_{n=-\infty}^{n=+\infty}(-1)^{n} x^{3\left(3 n^{2}+n\right)}
$$

is an example of the general theorem for the sequence of odd integers and all integers respectively in order, viz. :

$$
\left(p_{1}, p_{2}, \ldots, p_{r}, \ldots\right)=(1,1,3,2,5,3, \ldots, 2 r-1, r, \ldots) .
$$

The form of the theorem then is

$$
\begin{aligned}
f(x)=f(0) & +f^{(1)}(0) x+f^{(2)}(0) \frac{x^{2}}{2}+f^{(3)}(0) \frac{x^{5}}{20}+\ldots \\
& +f^{(2 r-1)}(0) \frac{2^{r}}{6 r-4.6 r-7 \ldots 5^{\cdot} 2} x^{\frac{3}{\left(3 r^{3}-r\right)}} \\
& +f^{(2 r)}(0) \frac{2^{r}}{6 r-2.6 r-5 \ldots 4 \cdot 1} x^{3\left(3 r^{2}+r\right)}+\ldots
\end{aligned}
$$

The forms for the sequences $(2,2,2, \ldots)$ and $(1,2,2,2, \ldots)$ are respectively

$$
\begin{aligned}
& f(x)=f(0)+f^{(1)}(0) x^{2}+f^{(2)}(0) \frac{x^{4}}{2 !}+\ldots+f^{(m)}(0) \frac{x^{2 m}}{m !}+\ldots \\
& f(x)=f(0)+f^{(1)}(0) x+f^{(2)}(0) \frac{x^{3}}{3}+\ldots+f^{(m)}(0) \frac{x^{2 m-1}}{(m-1) !(2 m-1)}+\ldots
\end{aligned}
$$

These are the forms of the theorem for even functions and odd functions respectively. Comparing the coefficients of these series with the coefficients of the normal form of Maclaurin's series, we see that for even functions

while for odd functions

$$
f^{(m)}(0)=\frac{m !}{2 m !} f^{2 m}(0)
$$

$$
f^{(m)}(0)=\frac{(m-1) !}{(2 m-2) !} f^{2 m-1}(0) .
$$

* "Series connected with the Enumeration of Partitions," supra, pp. 84 et seq.; Trans. R.S. Edin., "Generalized Functions of Legendre and Bessel," Vol. xl1.; "Extension of Neumano's Theorem," infia, pp. 361 et seq. ; Proc. R.S. Edin., Vol. xxur., pp. 440-447. 
In (17) $f^{(m)}(x)=\left(\frac{d}{d\left(x^{2}\right)}\right)^{m} f(x)$, but in (18) $f^{(m)}(x)=\left(\frac{d}{d\left(x^{2}\right)}\right)^{m-1}\left(\frac{d}{d x}\right) f(x)$.

The fundamental hypergeometric series and its differential equation are discussed in a paper (Trans. R. S. Edin., Vol. xuI., p. 29). This series is of type (1), being

$$
F\left[\left(\alpha_{1}\right)\left(\alpha_{2}\right)\left(\alpha_{3}\right) \ldots\left(\alpha_{n}\right)\left(\beta_{1}\right)\left(\beta_{2}\right) \ldots\left(\beta_{m}\right) x\right]=\Sigma A_{r} x^{p_{1}+p_{2}+\ldots+p_{r}}=\Sigma A_{r} x^{(r)},
$$

in which

$$
\begin{aligned}
A_{r}=p_{1}^{r}\left[\left\{\left[\left(\alpha_{1}\right)\left(\alpha_{1}+p_{1}\right)\left(\alpha_{1}+p_{1}+p_{2}\right) \ldots\left(\alpha_{1}+p_{1}+p_{2}+\ldots+p_{r-1}\right)\right]\right.\right. \\
\left.\quad \times\left[(n-1) \text { similar products for } \alpha_{2}, \alpha_{3}, \ldots, \alpha_{n}\right]\right\} \\
\div\left\{\left[\left(p_{1}\right)\left(p_{1}+p_{2}\right)\left(p_{1}+p_{2}+p_{3}\right) \ldots\left(p_{1}+p_{2}+\ldots+p_{r}\right)\right]\right. \\
\quad \times\left[\left(\beta_{1}\right)\left(\beta_{1}+p_{2}\right)\left(\beta_{1}+p_{2}+p_{9}\right) \ldots\left(\beta_{1}+p_{2}+p_{3}+\ldots+p_{r}\right)\right] \\
\left.\left.\quad \times\left[(m-1) \text { similar products for } \beta_{2}, \ldots, \beta_{m}\right]\right\}\right]
\end{aligned}
$$

For the sequence $(1,1,1, \ldots)$ this reduces to the hypergeometric series with $n$ elements in the numerator, and $m$ elements in the denominator of each term. 